\title{
Multiresolution Interpolation and Detiding of the ADCP Data
}

\author{
LING-YUN CHIAO \\ Institute of Oceanography, National Taiwan University, Taipei, Taiwan \\ YU-HUAI WANG \\ National Center for Ocean Research, Taipei, Taiwan
}

(Manuscript received 17 December 2001, in final form 19 May 2003)

\section{ABSTRACT}

\begin{abstract}
To account for the uneven sampling of current measurements collected by acoustic Doppler current profilers (ADCPs), a robust, three-dimensional interpolation scheme based on the multiresolution representation of the regional mean and tidal current fields is proposed. Instead of reconstructing the tidal field by getting bin-averaged time series that rely on heavy sampling or invoking radial basis function expansions, such as using the biharmonic splines with subjectively selected knots, the resolving capability of the proposed scheme relies fundamentally on the scale hierarchy of the resolvable local information constrained by the data. It is demonstrated that the proposed scheme flexibly incorporates the merits of the two conventional techniques. It enforces the resolution of model information while accommodating the local sampling density. Since it is based on a knots network defined by regular grids, attempts at experimentally and subjectively constructing the proper number and locations of controlling nodes are avoided. Constructing multiresolution representation of the current fields in terms of the three-dimensional wavelet basis is implemented by the computationally effective discrete wavelet transform of coefficients of the interpolation equations. Applications of the proposed multiresolution scheme on artificial as well as field datasets of the ADCP measurements demonstrate that it is a promising approach.
\end{abstract}

\section{Introduction}

The acoustic Doppler current profiler (ADCP) has become an indispensable tool for ocean current measurements in recent years. However, uneven sampling in both space and time has rendered the systematic processing of ADCP measurements difficult and diminished its potential impact on the effective mapping of ocean currents. Fortunately, the dominant transient variations of currents are usually composed of tidal variations with known periods. Separating the transient signal and interpreting the spatial pattern of the regional current field based on the ADCP measurements thus rely on robustly reconstructing the spatially continuous variations representing the tidal fields. One method is to build a barotropic or even a baroclinic numerical model of the tides for the specific study region as the reference model for detiding the ADCP data (Foreman and Freeland 1991). Assimilation of the ADCP data into the barotropic model (Dowd and Thompson 1996; Bogden and O'Donnell 1998) makes use of the information from the field data yet demands extensive a priori knowledge of the local tidal fields under study.

Corresponding author address: Ling-Yun Chiao, Institute of Oceanography, National Taiwan University, Taipei, Taiwan. E-mail: chiao@ccms.ntu.edu.tw
Alternatively, the second school of techniques developed for detiding the ADCP data attributes the transient variation to known periods of the regional tides and these techniques are essentially different schemes of spatial interpolation. This may include straightforward segmenting of the ship track into horizontal bins and constructing the time series, by ignoring spatial variation, within a bin-for-bin harmonic analysis (Simpson et al. 1990). Sites without adequate sampling of the transient variation to construct the time series have to be interpolated from nearby bins with available information (Geyer and Signell 1990). Candela et al. (1990, 1992), on the other hand, invoke polynomials and the biharmonic splines as interpolating functions to expand the spatial variations of the tidal fields. Although these expansions have been implemented to depict lateral variation utilizing the depth-averaged current measurements, Münchow (2000) generalized the technique to the three-dimensional space in a current velocity survey in coastal waters. Since these exploited interpolating functions are of fixed spatial forms, the derivation of the optimal number and locations of where these a priori functions should be located within the study region becomes extremely critical. The locations are usually selected subjectively.

Sampling density of the ADCP data in both space 
and time is usually highly heterogeneous. It is thus unrealistic to expect that the scale spectrum of the resolvable components will be uniform throughout the study region. This important aspect of the interpolation and detiding of the ADCP data has not been explored and is one of the major motivations for the present study. We believe that the well-developed multiresolution representation of a function (Mallat 1989a,b) has merits for devising a data-adaptive interpolation scheme without tedious efforts of pinning the controlling nodes. We will briefly review the methods of interpolating the ADCP data and develop an alternative scheme of the multiresolution parameterization based on the three-dimensional wavelet basis functions.

\section{Method}

\section{a. Bin averaging and radial basis function expansion}

Based on the presumption that the dominant transient variations of coastal currents are composed of tidal variations with known periods, each of the components of the current velocity vector field measured by the ADCP is represented by

$$
\begin{aligned}
u\left(\mathbf{r}_{i}, t_{i}\right)=u_{0}\left(\mathbf{r}_{i}\right)+\sum_{j=1}^{M} & {\left[b_{j}\left(\mathbf{r}_{i}\right) \cos \left(\omega_{j} t_{i}\right)\right.} \\
& \left.+c_{j}\left(\mathbf{r}_{i}\right) \sin \left(\omega_{j} t_{i}\right)\right]+\varepsilon_{i} .
\end{aligned}
$$

That is, for the $i$ th data $u_{i}=u\left(\mathbf{r}_{i}, t_{i}\right), \mathbf{r}_{i}=\left(x_{i}, y_{i}, z_{i}\right)^{\mathrm{T}}$ and $t_{i}$ are the position and time the measurement is made; $\varepsilon_{i}$ is the measurement noise or any component not accounted for by the mean field $u_{0}$ and the $M$ invoked periods of tidal component $b_{j}, c_{j}$. It is clear that an implicit assumption embedded within (1) is that all of the transient variation is attributable to the tidal components. This might be true for a survey across a short duration of time, but it will be invalidated when attempting to combine historical datasets that might last over years. Under those circumstances, it is possible to add in extra terms to $u_{0}$, such as low-order polynomials of time, to compensate for the long period transient component in the mean field. However, the additional degrees of freedom would certainly downgrade the fitting unless heavy sampling is available. Once the types $\left(\mathrm{K}_{1}, \mathrm{M}_{2}, \ldots\right)$ and the number $(M)$ of periods are determined based on a priori information from other observations or experiences obtained from previous regional studies, the task is then to reconstruct the intrinsically continuous field $u_{0}, b_{j}, c_{j}(j=1, \ldots, M)$ based on the finite dataset $u_{i}(i=1, \ldots, N)$, which is usually distributed both sparsely and unevenly. One method is to sort data points into regular horizontal bins along ship tracks and construct time series for a particular bin assuming spatial variations are negligible within the bin. Harmonic analysis by least squares fitting to the obtained time series for each bin is then performed to separate the mean field and the tidal fields. In general, high sampling density is required to ensure that there are enough constraints, with adequate time coverage, within a particular bin for resolving the tidal components. Furthermore, there is a potential problem with space-time aliasing. Namely, if the width of a particular bin is chosen to be too narrow, there may not be enough sampling for a successful harmonic analysis to recover the tidal components robustly. On the other hand, a bin that is too wide might bear significant lateral variations that will be mapped into a transient variation of the tidal components obtained from a naïve harmonic analysis.

Another technique to detide the ADCP data (Candela et al. 1990, 1992) takes an approach that can be categorized as the radial basis function (RBF) expansion. A RBF expansion (e.g., Kirby 2001) of a continuous spatial function $f(\mathbf{r})$ is approximated by a finite set of degrees of freedom, $w_{k}$ :

$$
\tilde{f}(\mathbf{r})=\sum_{k=1}^{K} w_{k} \phi\left(\mathbf{r}, \mathbf{r}_{k}\right),
$$

where $\mathbf{r}_{k}$ denotes the particular knot location associated with the $k$ th $\operatorname{RBF} \phi\left(\mathbf{r}, \mathbf{r}_{k}\right)$. In the case of representing the ADCP data with the chosen RBF, the current velocity field, (1), is of the form

$$
\begin{aligned}
& u(\mathbf{r}, t)=\sum_{k=1} \alpha_{k} \phi\left(\mathbf{r}, \mathbf{r}_{k}\right) \\
&+\sum_{j=1}^{M}\left[\sum_{k^{\prime}} \beta_{j k^{\prime}} \cos \left(\omega_{j} t\right) \varphi\left(\mathbf{r}, \mathbf{r}_{k^{\prime}}\right)\right. \\
& \\
&\left.\quad+\sum_{k^{\prime \prime}} \gamma_{j k^{\prime \prime}} \sin \left(\omega_{j} t\right) \psi\left(\mathbf{r}, \mathbf{r}_{k^{\prime \prime}}\right)\right] .
\end{aligned}
$$

Although the number of knots, $k, k^{\prime}, k^{\prime \prime}$, and the RBFs, $\phi, \varphi, \psi$, can be different for the mean field and the tidal components, a considerably simplified expression can be cast as

$$
\begin{aligned}
u(\mathbf{r}, t)= & \sum_{k=1}^{K}\left\{\alpha_{k}+\sum_{j=1}^{M}\left[\beta_{j k} \cos \left(\omega_{j} t\right)+\gamma_{j k} \sin \left(\omega_{j} t\right)\right]\right\} \\
& \times \phi\left(\left|\mathbf{r}-\mathbf{r}_{k}\right|\right)
\end{aligned}
$$

Notice that the spatial dependence of the RBF is usually defined in terms of the distance, $\left|\mathbf{r}-\mathbf{r}_{k}\right|$, away from the knot location, $\mathbf{r}_{k}$. Expression (4) is fitted to the ADCP measurements in the least squares sense to determine the $K(2 M+1)$ coefficients $\alpha_{k}, \beta_{j k}, \gamma_{j k}$. That is, we seek coefficients that minimize the misfit $\sum_{i=1}^{N}$ $\left(u_{i}^{\text {obs }}-u_{i}^{\text {pre }}\right)^{2}$, where $u_{i}^{\text {obs }}$ is one of the $N$ current velocity observations and $u_{i}^{\text {pre }}$ is the velocity predicted by (4) at the time and place $u_{i}^{\text {obs }}$ is observed. Although there are various types of admissible RBFs (Kirby 2001, Table $7.1), \phi$ in (4), that perform differently, the biharmonic splines (Sandwell 1987) and polynomial functions have been very popular for the interpolation of ADCP data (e.g., Candela et al. 1990, 1992; Foreman and Freeland 1991; Münchow 2000). These RBFs can be categorized as being of a global type (Kirby 2001), since in con- 
straining the coefficients, $\alpha_{k}, \beta_{j k}, \gamma_{j k}$ of (4), data observed far away from a particular knot do not have decaying influences as compared to the nearby observations. It is interesting that other RBFs, such as a Gaussian function that behaves locally, have seldom been considered in interpolating the ADCP data. It is reported that the performance of these particular interpolating functions depends critically on the number and locations of the controlling nodes (or knots), that is, $K$ and $\mathbf{r}_{k}$ in (4). The main reason for this is that due to the transient variation, the ADCP observations at any instant are usually distributed highly unevenly throughout the study region, even though the ship tracks are usually carefully planned for a typical survey. Both of the number $K$ and the locations $\mathbf{r}_{k}$ have to be carefully designed for any chosen RBFs such that rich information in the well-sampled area is not wasted whereas unstable complexities, not robustly constrained by the data, will not be introduced into the constructed fields for a poorly constrained area. As a consequence, the process of selecting nodes is usually achieved empirically through tedious experiments, which depend on the subjective decisions of the practitioner (e.g., Münchow 2000).

\section{b. Regular gridding}

Instead of pinning a specially designed knots network, it is straightforward to construct a regular threedimensional tessellation with grid intervals $\Delta x, \Delta y, \Delta z$. That is, the $3 \mathrm{D}$ computation domain of $l_{x} \times l_{y} \times l_{z}$ is subdivided into $K=K_{x} \times K_{y} \times K_{z}$ pixels (or cubic cells), with $K_{x} \times \Delta x=l_{x}$, etc. Based on the regular grid and a chosen RBF, coefficients of (4) can be grouped into the following $K$-dimensional vectors: $\boldsymbol{\alpha}$, $\boldsymbol{\beta}_{j}, \boldsymbol{\gamma}_{j}$ with elements $\alpha_{l m n}$, where $l=1, \ldots, K_{x}, m=$ $1, \ldots, K_{y}, n=1, \ldots, K_{z}$, for the mean field $u_{0}(\mathbf{r})$; and $\beta_{j l m n}, \gamma_{j l m n}$ for each of the invoked harmonics, $b_{j}(\mathbf{r})$, $c_{j}(\mathbf{r}), j=1, \ldots, M$, as in Eq. (1). Equation (4) can be reduced to

$$
\begin{aligned}
& u_{i}=\mathbf{a}_{i} \cdot \boldsymbol{\alpha}+\sum_{j=1}^{M}\left[\mathbf{b}_{i j} \cdot \boldsymbol{\beta}_{j}+\mathbf{c}_{i j} \cdot \boldsymbol{\gamma}_{j}\right] \text { or } \\
& u_{i}=G_{i j} m_{j},
\end{aligned}
$$

$\left[\begin{array}{c}\boldsymbol{\alpha} \\ \boldsymbol{\beta}_{1} \\ \boldsymbol{\gamma}_{1} \\ \boldsymbol{\beta}_{2} \\ \boldsymbol{\gamma}_{2} \\ \vdots\end{array}\right), \quad \mathbf{G}_{i}=\left[\begin{array}{llllll}\mathbf{a}_{i}^{\mathrm{T}} & \mathbf{b}_{i 1}^{\mathrm{T}} & \mathbf{c}_{i 1}^{\mathrm{T}} & \mathbf{b}_{i 2}^{\mathrm{T}} & \mathbf{c}_{i 2}^{\mathrm{T}} & \cdots\end{array}\right]$,

where $u_{i}=u\left(\mathbf{r}_{i}, t_{i}\right)$ is the $i$ th ADCP measurement observed at time $t_{i}$ and location $\mathbf{r}_{i}$, and transposes of column vectors $\mathbf{a}_{i}^{\mathrm{T}}=\left[\phi\left(\left|\mathbf{r}_{i}-\mathbf{r}_{1}\right|\right) \phi\left(\left|\mathbf{r}_{i}-\mathbf{r}_{2}\right|\right) \cdots \phi\left(\mid \mathbf{r}_{i}\right.\right.$ $\left.\left.-\mathbf{r}_{K} \mid\right)\right], \mathbf{b}_{i j}^{\mathrm{T}}=\left[\phi\left(\left|\mathbf{r}_{i}-\mathbf{r}_{1}\right|\right) \cos \left(w_{j} t_{i}\right) \phi\left(\left|\mathbf{r}_{i}-\mathbf{r}_{2}\right|\right)\right.$ $\left.\cos \left(w_{j} t_{i}\right) \cdots \phi\left(\left|\mathbf{r}_{i}-\mathbf{r}_{K}\right|\right) \cos \left(w_{j} t_{i}\right)\right]=\cos \left(w_{j} t_{i}\right) \mathbf{a}_{i}^{\mathrm{T}}$, and $\mathbf{c}_{i j}^{\mathrm{T}}=\sin \left(w_{j} t_{i}\right) \mathbf{a}_{i}^{\mathrm{T}}$ are elements of the $\mathbf{G}$ matrix constraining the model parameter vector $\mathbf{m}$. As mentioned earlier, popular RBFs for the interpolation of the ADCP data such as the polynomials and the biharmonic splines tend to behave globally. One advantage of invoking a localtype RBF such as a Gaussian function is that the $\mathbf{G}$ matrix in (6) will be very sparse, since influences from faraway knots decay rapidly with increasing distances for a particular observation. It is well known that a sparse matrix is numerically much easier to solve than a full matrix (e.g., Paige and Saunders 1982; Press et al. 1992). Physically, it also makes sense to have the interpolation of the current speed behave locally; that is, the interpolation at a particular site should not depend on constraints very far away. An example of such a local-type RBF can be a boxcar function:

$$
\phi\left(\left|\mathbf{r}-\mathbf{r}_{k}\right|\right)=\left\{\begin{array}{cc}
1, & \left|x-x_{k}\right| \leq \Delta x / 2, \\
& \left|y-y_{k}\right| \leq \Delta y / 2, \\
& \left|z-z_{k}\right| \leq \Delta z / 2 \\
0, & \text { otherwise, }
\end{array}\right.
$$

where $\mathbf{r}=\left(\begin{array}{lll}x & y & z\end{array}\right)^{\mathrm{T}}$ and $\mathbf{r}_{k}=\left(\begin{array}{lll}x_{k} & y_{k} & z_{k}\end{array}\right)^{\mathrm{T}}$. Equations (5) and (6) then take the form

$$
\begin{aligned}
u_{i}=\delta_{p l} \delta_{q m} \delta_{r n}\left\{\alpha_{l m n}+\sum_{j=1}^{M}[\right. & \beta_{j l m n} \cos \left(\omega_{j} t_{i}\right) \\
& \left.\left.+\gamma_{j l m n} \sin \left(\omega_{j} t_{i}\right)\right]\right\},
\end{aligned}
$$

where $\left\|x_{i}-p \Delta x\right\| \leq \Delta x / 2,\left\|y_{i}-q \Delta y\right\| \leq \Delta y / 2, \| z_{i}-$ $r \Delta z \| \leq \Delta z / 2$.

To obtain model parameter vector $\mathbf{m}$ by solving Eq. (8) in the least squares sense will be very similar to executing the bin averaging within a cell that has adequate observations. In general, the $\mathbf{G}$ matrix in (6) is very likely to be singular, since fields of the undersampled pixels are not resolvable. Numerically, we have to regularize the potentially ill-posed system (e.g., Tikhonov and Goncharsky 1987), or invoke the damped least squares method (DLS; e.g., Lines and Treitel 1983) rather than the straightforward least squares fitting. This is done by minimizing a weighted sum of the misfit and the model norm, $\left\|\mathbf{u}^{\text {obs }}-\mathbf{u}^{\text {obs }}\right\|+\lambda\|\mathbf{m}\|$, rather than the misfit only. In the above expression, $\|\cdot\|$ denotes the $L_{2}$ norm of a vector and the damping factor $\lambda$ controls the relative rigor of the regularization (or damping). The value $\lambda$ is usually determined by the variance-reduction versus model-variance trade-off analysis. Briefly, the variance reduction is defined to indicate the capability of an obtained model (m) to reconstruct the observed data (d). It can be calculated by

$$
v_{r}=\left(1-\frac{\|\mathbf{G m}-\mathbf{d}\|^{2}}{\|\mathbf{d}\|^{2}}\right) \times 100 \% .
$$

On the other hand, the model variance is a measure of 
the uncertainty of a model obtained from noisy data; it is computed (Paige and Saunders 1982) by

$$
\begin{aligned}
\sigma_{m} & =\sum_{l=1}^{K(2 M+1)} s_{l}^{2} \\
s_{l}^{2} & =\|\mathbf{d}-\mathbf{G m}\|^{2} \sigma_{l l}, \quad \sigma_{l l}=\operatorname{diag}\left[\left(\mathbf{G}^{\mathrm{T}} \mathbf{G}+\lambda^{2} \mathbf{I}\right)^{-1}\right] .
\end{aligned}
$$

It is noted that heavier damping set up by a large value of $\lambda$ usually leads to a robust model (lower $\sigma_{m}$ ), but sacrifices the data fitting (lower $v_{r}$ ) at the same time. We will show in the applications below how the tradeoff between model robustness and data fitting helps to determine an appropriate $\lambda$ and an optimal model.

The regularized solution of (8) is numerically stable but yields negligible amplitude for undersampled pixels. One method of improving this situation is to simply enlarge the extends of these undersampled pixels such that enough constraints can be accumulated. This will be analogous to techniques used in variable cell parameterizations, which have been implemented in modern seismic tomography (Bijwarrd et al. 1998; Gualtiero and Vesnaver 1999), or imposing regularization criteria of model smoothness instead of model norm (e.g., Meyerholtz et al. 1989). The latter approach is effectively analogous to letting the RBFs, $\phi\left(\left|\mathbf{r}-\mathbf{r}_{k}\right|\right)$ invoked in the ADCP interpolation, have lengthened influences across neighboring pixels so that constraints farther away can be taken advantage of. This can be achieved, for example, by having wider characteristic correlation lengths $\sigma_{x}, \sigma_{y}, \sigma_{z}$ in a Gaussian-type RBF of the form $A \exp \left\langle-0.5\left\{\left[\left(x-x_{k}\right)^{2}\right] / \sigma_{x}^{2}+\left[\left(y-y_{k}\right)^{2}\right] / \sigma_{y}^{2}+[(z-\right.\right.$ $\left.\left.\left.\left.z_{k}\right)^{2}\right] / \sigma_{z}^{2}\right\}\right\rangle$. In both of these two approaches, an efficient scheme of scanning through the spatially heterogeneous distribution of the ADCP observations at a particular time has to be implemented. The intension is to differentiate the well-sampled and the poorly sampled areas and then modify either the geometric configurations of the grids or the RBFs of those poorly sampled areas. Without such a scheme, these modifications would be carried out subjectively, just like the conventional knots planning. Furthermore, variations across time embedded in the ADCP measurements complicate the process and potentially render the operation of grouping nearby pixels impractical.

To briefly summarize, we have built a 3D knots network based upon regular grid intervals for the ADCP interpolation instead of subjectively pinning knots within the study region. It is obvious that some of the knots are very likely to be without appropriate data constraints. Consequently, the resulting matrix equation has to be regularized. For a regular grid, a least squares solution of the ADCP interpolation obtained by the model norm regularization is analogous to the conventional bin-averaging results. On the other hand, regularization criteria based on the model smoothness leads to results similar to these for least squares solutions for

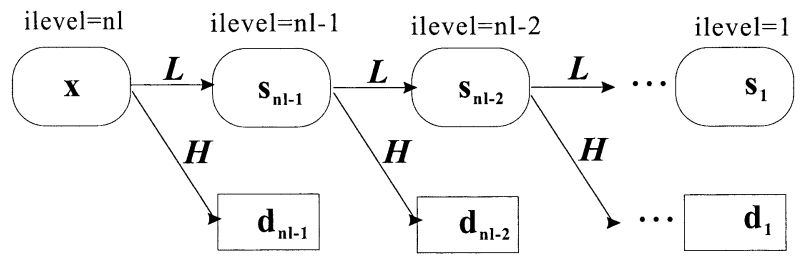

FIG. 1. Pyramid algorithm (Mallat 1989a) that performs a fast wavelet transform or the multiresolution decomposition of a discrete signal $\mathbf{x}$. Here, $L, H$ are low-pass and high-pass filters corresponding to a particular chosen wavelet basis function. Convolution of the smooth signal $\mathbf{s}_{i}$ at the $i$ th level with $L$ and $H$ generates the smooth signal $\mathbf{s}_{i-1}$ and the detail $\mathbf{d}_{i-1}$ at the next lower and coarser level while the characteristic scale length is doubled.

an RBF expansion with long correlation length. We will introduce our proposed multiresolution approach in the following section and demonstrate that it flexibly incorporates the merits of these two conventional techniques. These three different approaches will be further compared for applications on real and synthetic datasets.

\section{c. Multiresolution representation}

A tessellation created with regular grids avoids subjective decisions made on the locations of the controlling knots. However, as just discussed in the previous section, intrinsically heterogeneous sampling of the ADCP data downplays this advantage unless a dataadaptive reorganizing scheme is at hand. This scheme should enable the resolution of details of the current fields for a densely sampled area, but also conservatively preserve the robust resolution of large-scale structures for regions with fewer available constraints. In the following, we will first introduce, very briefly, a simple example of the discrete wavelet transform (DWT) and show that the corresponding concept of multiresolution representation proposed by Mallat (1989a,b) helps to devise such a scheme (see also Chiao and Kuo 2001; Chiao and Liang 2003).

Without loss of generality, we take a general 1D time series as an example to help summarize the essence of the DWT. The most straightforward generalization to higher dimensions can be achieved by direct tensor products (Press et al. 1992; Mallat 1998). For the 1D time series $\mathbf{x}=\left(x_{1}, x_{2}, \ldots x_{i}, \ldots x_{N}\right)^{\mathrm{T}}$ sampled at very $\Delta t$, the DWT (e.g., Bruce and Gao 1996; Mallat 1998) can be undertaken by the numerically efficient pyramid algorithm (Mallat 1989b), which decomposes the time series into a low-passed signal and a high-passed detail successively at each level with increasing scale lengths (Fig. 1). Specifically, if we choose to construct the highpass filter by the simple Haar wavelet basis, then starting from the original signal $\mathbf{x}$ with scale length $\Delta t$, designated to be at the highest scale level $(n l)$ or the leaves level, we can generate the low-passed signal $\mathbf{s}_{n l-1}$ and the high-passed signal $\mathbf{d}_{n l-1}$ at the lower scale level ( $n l$ - 1) by 


\section{(a) along-shore}

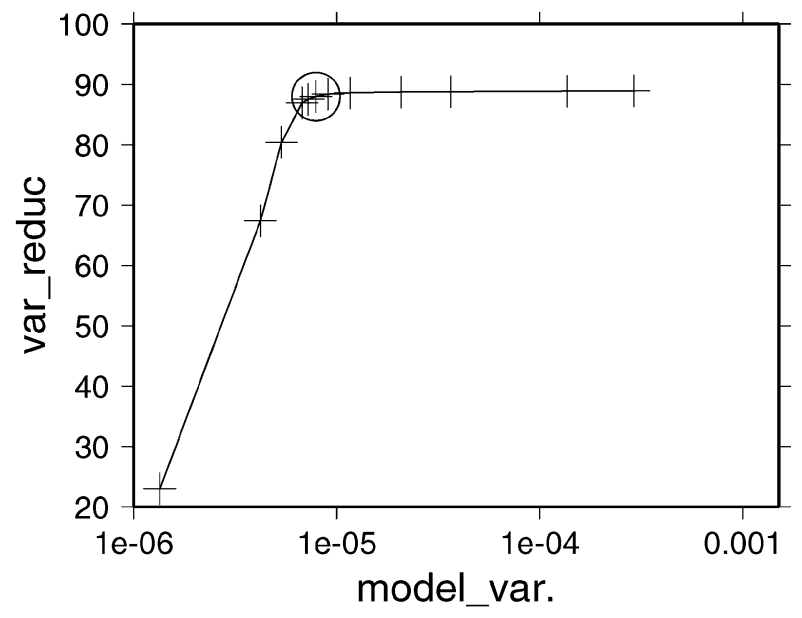

(b) across-shore

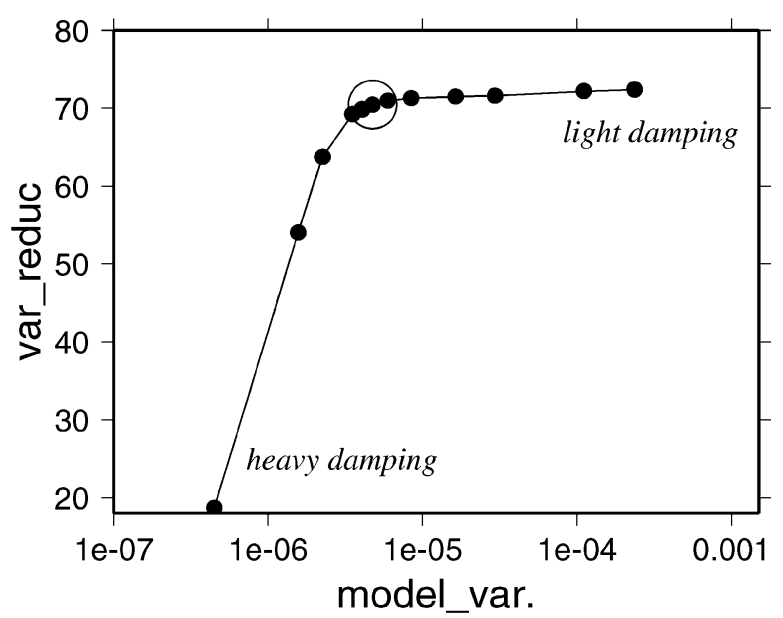

FIG. 2. Trade-off curves of the multiresolution interpolation of (top) the $v$ component, which is in the alongshore direction, and (bottom) the $u$ component, which is normal to the coast in the off-shore area near the outlet of the Tamshui River. The vertical axis is marked by percentage of variance reduction [Eq. (9)], which is an integrated measure of the data fitting. Values on the horizontal axis, in logarithm, stand for model variance defined by (10), which is a global measure of the model uncertainty, manifested from the data noise, of the reconstructed flow fields.

$$
\begin{array}{r}
\mathbf{s}_{n l-1}=\left[s_{n l-1,1}=\left(x_{2}+x_{1}\right) / 2, \ldots,\right. \\
\left.s_{n l-1,2^{n l-2}}=\left(x_{N}+x_{N-1}\right) / 2\right] \\
\mathbf{d}_{n l-1}=\left[d_{n l-1,1}=\left(x_{2}-x_{1}\right) / 2, \ldots,\right. \\
\left.d_{n l-1,2^{n l-2}}=\left(x_{N}-x_{N-1}\right) / 2\right] .
\end{array}
$$

In other words, the low-pass filtering for this particular wavelet basis is simply taking the algebraic average of every neighboring pair. This operation generates the signal $\mathbf{S}_{n l-1}$ as a realization of the original time series at a lower scale level $(n l-1)$ with a lengthened scale of
$2 \Delta t$. To be able to restore the original signal $\mathbf{x}$, we also have the high-passed detail signal $\mathbf{d}_{n l-1}$ at the same scale level. The original $N$ degrees of freedom is thus split equally into both the smooth signal $\mathbf{s}_{n l-1}$ and the detail $\mathbf{d}_{n l-1}$ at a lower scale level. The signal can be further decomposed successively into $\mathbf{s}_{n l-2}$ and $\mathbf{d}_{n l-2}$ at an even lower scale level $(n l-2)$ and a larger scale until the lowest level, also known as the root level, is reached where $\mathbf{s}_{1}$ and $\mathbf{d}_{1}$ are generated. The result of the accomplished transform of $\mathbf{x}$ yields

$$
\begin{aligned}
& \mathbf{y}=W(\mathbf{x}),
\end{aligned}
$$

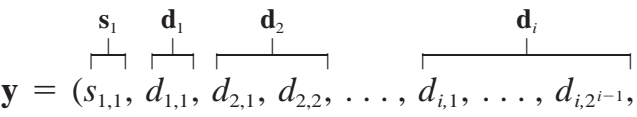

$$
\begin{aligned}
& \frac{\mathbf{d}_{n l}}{\left.d_{n l, 1}, \ldots, d_{n l, 2^{n l-1}}\right)^{\mathrm{T}}},
\end{aligned}
$$

where $\mathbf{x}$ is the original realization with the characteristic scale $\Delta t ; \mathbf{s}_{1}$ is the signal realized at scale length $N \Delta t$ and $\mathbf{d}_{i}$ is the detail signals at a scale length in between $\Delta t$ and $N \Delta t$. That is, $\mathbf{y}$ consists of the basic smooth signal $\mathbf{s}_{1}$ at the root level that has the long characteristic scale $N \Delta t$, and all the details at successively shorter scales. The reason that this is a multiresolution representation is due to the fact that we can always construct the signal realized at all different scales. For example, combining $\mathbf{s}_{1}$ and $\mathbf{d}_{1}$ reconstructs $\mathbf{s}_{2}$, and successively blending in details at shorter scale generates $\mathbf{s}_{i}$, which would eventually rebuild the original signal fully at the highest resolution. This reconstruction is essentially the inverse of the wavelet transform:

$$
\mathbf{x}=W^{-1}(\mathbf{y}) \text {. }
$$

More general wavelet bases other than the Haar wavelet have been widely utilized to build the low-pass and the high-pass filters (e.g., Mallat 1998). These wavelet bases are mutually orthogonal across different scale levels whereas the low-pass basis and the high-pass basis at the same level are also orthogonal to each other. However, the latter requirement has been relaxed in order to have a symmetric basis without skewed filtering (Cohen et al. 1992). It leads to the development of the family of biorthogonal wavelets (Cohen et al. 1992; Mallat 1998). A wavelet transform utilizing the biorthogonal wavelet bases has two complementary bases sets at each scale level. One set is named the primary basis and the other is the dual basis. The low-pass basis and highpass basis at the same scale level from each of the same bases set are not mutually orthogonal. However, bases from the primary set are always orthogonal to bases from the dual set. It is customary to denote the wavelet transform utilizing the primary wavelet basis by $W(\mathbf{x})$, and the wavelet transform utilizing the dual basis by $W^{*}(\mathbf{x})$ (e.g., Bruce and Gao 1996). Due to the biorthogonality, any inner product between two signals $\mathbf{x}_{1}$ and $\mathbf{x}_{2}$ will have 
(a) sampling

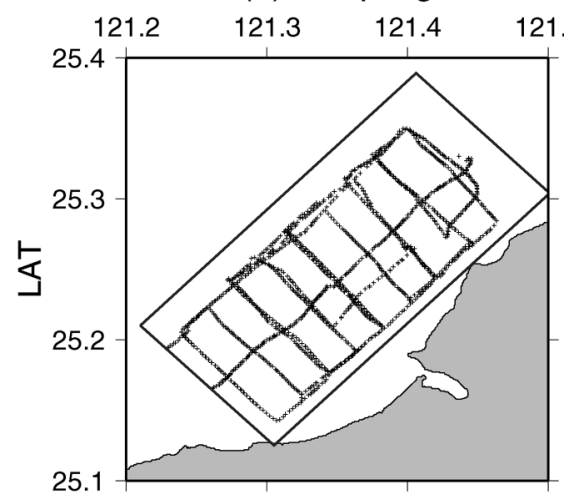

25.1 (c) M2

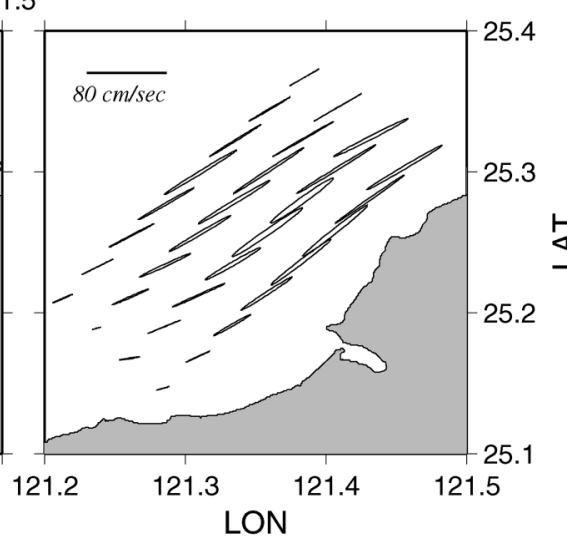

(b) mean flow

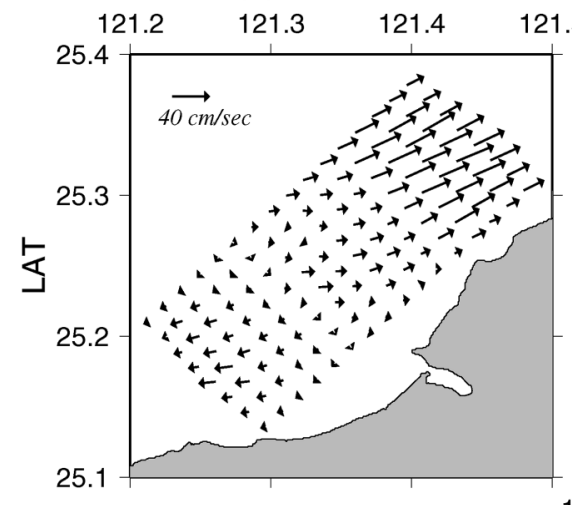

(d) K1

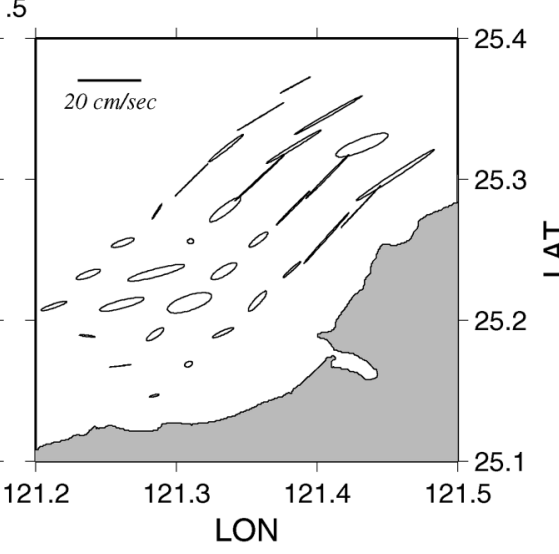

FIG. 3. (a) Map view of the spatial sampling of the ADCP measurements. The thin frame demarcates the interpolation region. (b) Multiresolutionally interpolated mean flow of a vertical layer centered at a depth around $25 \mathrm{~m}$. (c) Tidal ellipses of M2 of the same layer. (d) Tidal ellipses of $\mathrm{K} 1$ of the same layer.

$$
\mathbf{x}_{1} \cdot \mathbf{x}_{2}=W^{*}\left(\mathbf{x}_{1}\right) \cdot W\left(\mathbf{x}_{2}\right)=W\left(\mathbf{x}_{1}\right) \cdot W^{*}\left(\mathbf{x}_{2}\right) .
$$

In the formulation (5), we solve (6) to obtain coefficients $\boldsymbol{\alpha}$ of the mean field, as well as other tidal components $\boldsymbol{\beta}_{j}, \boldsymbol{\gamma}_{j}$, on pixels defined by regular grids. Instead of solving for $\boldsymbol{\alpha}$ directly, it is plausible to solve a matrix equation constraining the multiresolution representation $W(\boldsymbol{\alpha})$. The advantage of doing this is that there is a natural scale hierarchy embedded within $W(\boldsymbol{\alpha})$ due to the multiresolution representation. This enables resolving large-scale structures robustly, when solving the matrix equation constraining $W(\boldsymbol{\alpha})$, and gradually moves up the hierarchy for short-scale details depending on the local sampling. The underlying reason is that large-scale components are always constrained by more observations. To formulate the matrix equation for $W(\boldsymbol{\alpha})$, we take advantage of (14) and rewrite (5) as $u_{i}=W^{*}\left(\mathbf{a}_{i}\right) \cdot W(\boldsymbol{\alpha})$

$$
+\sum_{j=1}^{M}\left[W^{*}\left(\mathbf{b}_{i j}\right) \cdot W\left(\boldsymbol{\beta}_{j}\right)+W^{*}\left(\mathbf{c}_{i j}\right) \cdot W\left(\boldsymbol{\gamma}_{j}\right)\right] .
$$

Thus, instead of reformulating the interpolation equation using the wavelet bases, a direct wavelet transform of each row of the $\mathbf{G}$ matrix in (6), such as $W^{*}\left(\mathbf{G}_{i}\right)$, would compose the new matrix constraining $W(\mathbf{m})$. The solution can then always be inverse transformed to obtain the mean field as well as the tidal components, at different scale levels, hidden in the ADCP data. In short, since neither the time step nor the spatial sampling interval of the ADCP data is uniform, it is not possible to perform the usual DWT on the ADCP data directly. Instead, we take advantage of (15) and solve the reconfigured $\mathbf{G}$ matrix with rows $W^{*}\left(\mathbf{G}_{i}\right)$ to obtain $W(\mathbf{m})$, the wavelet transformed mean and tidal fields.

\section{Applications to field data and test example \\ a. Field data}

The Tamshui River is a major river system in northern Taiwan. Its outlet into the Taiwan Strait has been an important focus of research in recent years. We executed 
(a) surface

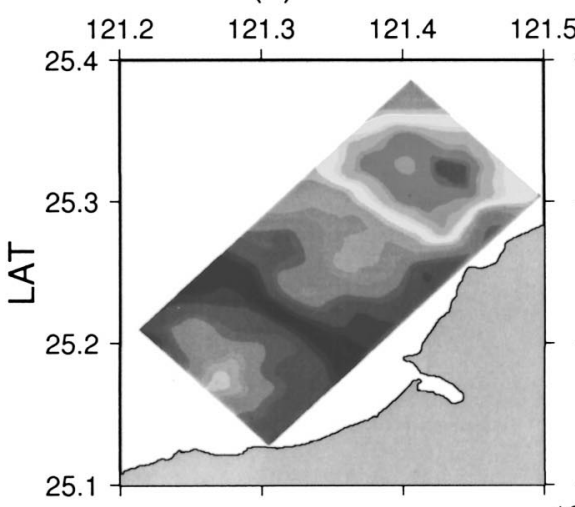

(c) Bin-averaging

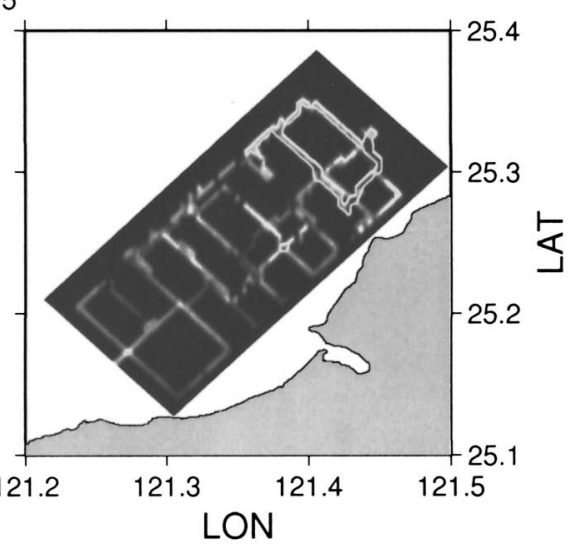

(b) depth $=60 \mathrm{~m}$

(d) Gaussian RBF expansion

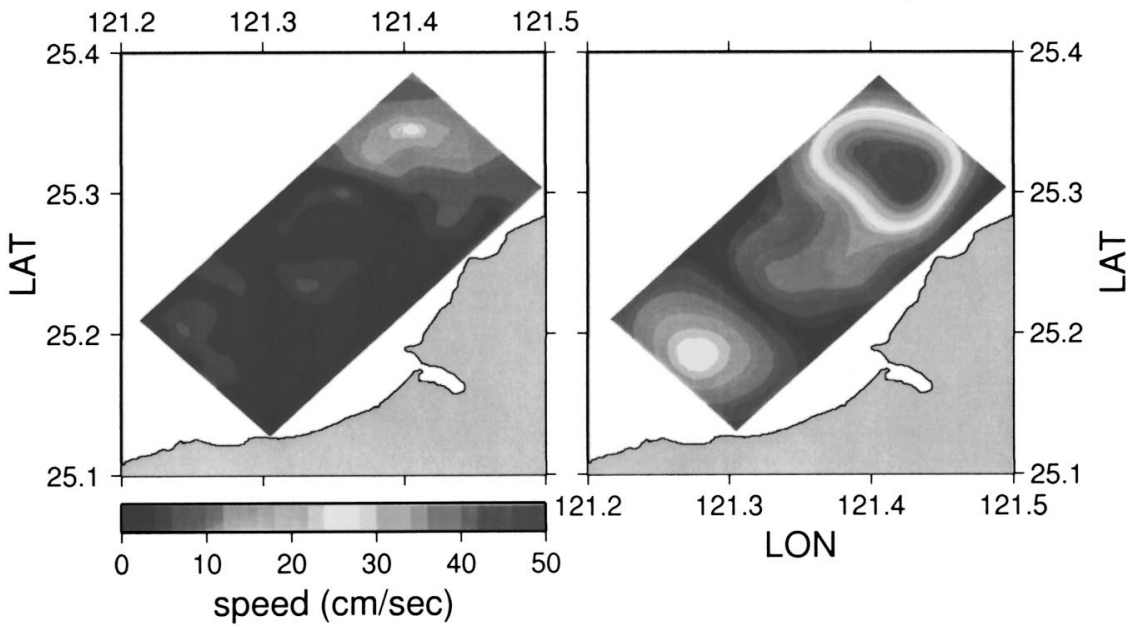

FIG. 4. Vertical variation of the mean current speed of the results obtained utilizing the proposed multiresolution parameterization: (a) the surface layer (from 0 to $15 \mathrm{~m}$ ) and (b) the bottom layer (from 45 to $60 \mathrm{~m}$ ). Also shown are the speed contours of the surface mean current obtained from a different method: (c) pixel-based DLS solution of fitting Eq. (8) to the observed data. This is in essence similar to the conventional bin-averaging scheme that lacks the capability of spatial interpolation. (d) RBF expansion invoking the Gaussian-type RBF with the correlation lengths defined to cross over neighboring pixels that are within three grid intervals in the three spatial directions, respectively.

a combined survey using three different ADCPs during 1-3 July 2001 to study the spatial patterns of the current field in this area. The entire survey covers only $36 \mathrm{~h}$, about 3 semidiurnal or 1.5 diurnal periods. The hullmounted 75-kHz RDI ADCP provides robust sampling with relatively low vertical resolution whereas measurements made by a Sontek $500-\mathrm{kHz}$ side-mounted and an RDI $600-\mathrm{kHz}$ towed instrument reveal high vertical resolution but appear to yield noisier measurements. The towing catamaran is designed to operate stably in wavy water with a towing speed of $6 \mathrm{kt}$. The bin depth is set to $1-4 \mathrm{~m}$ and the average time interval is $1-4 \mathrm{~min}$. The collected data are screened to remove outliers. The criteria are set such that the acceptable data are characterized by a percentage valid reading above $85 \%$, error velocity and vertical velocity of less than $15 \mathrm{~cm} \mathrm{~s}^{-1}$, ship speed at $50 \sim 300 \mathrm{~cm} \mathrm{~s}^{-1}$, and a change in heading of less than $2^{\circ} \mathrm{min}^{-1}$. Comparison among measurements obtained from the three different ADCPs suggests that the vertical averaged flows at a site measured by the three different profilers are almost identical. For example, the cross-correlation coefficients between the towed ADCP and the hull-mounted ADCP are 0.98 and 0.97 for the $u$ and $v$ components, whereas the corresponding root-mean-square errors are 10 and $8 \mathrm{~cm} \mathrm{~s}^{-1}$, respectively. The calibrated data amount to a set of 17000 velocity measurements. The interpolation carried out on the field data to map the coastal currents reveals some very interesting features.

As discussed earlier, three-dimensional tessellation is 

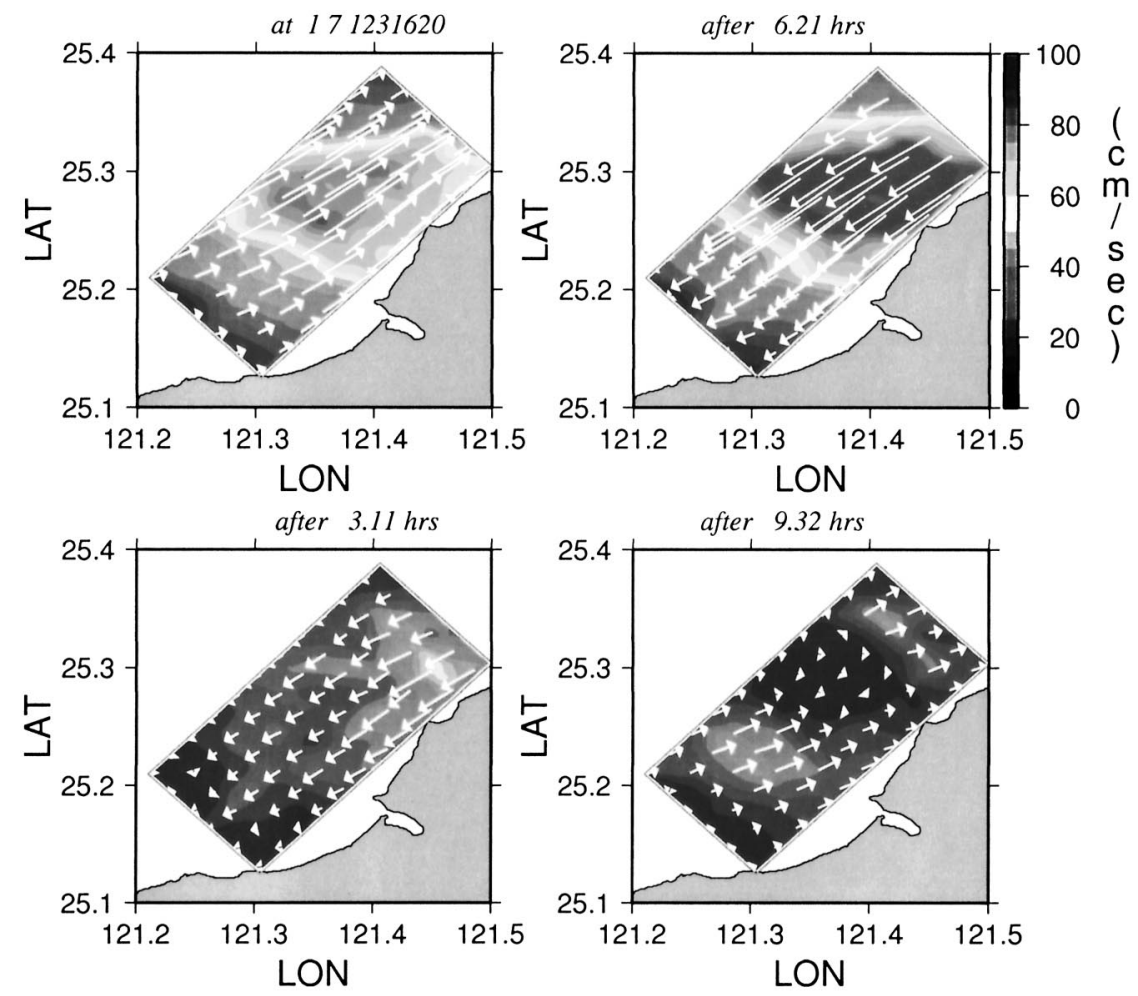

FIG. 5. Evolution of the tidal flow (both of the M2 and K1 combined) across a period of $T=12.42 \mathrm{~h}$. (top-left panel) The snapshot is first arbitrarily set at the initial time of the survey, and subsequently displayed at $t / T=\lambda / 2, \lambda, 3 \lambda / 2$.

first constructed upon the surveyed coast area near the outlet of the Tamshui River by $32 \times 64 \times 4$ cells. The size of the cell is about $420 \mathrm{~m}$ in both directions normal to and along the coastline, whereas the layer thickness is about $15 \mathrm{~m}$ in the vertical direction. The data equations describing each measurement, (5), are reconfigured according to (15) such that the solution will be the multiresolution representation of the mean and tidal flows. Several inversions with different damping factors are executed to construct the trade-off curve (Fig. 2). The optimal solution (circled in Fig. 2) is obtained by tuning the damping factor along the trade-off curve such that a solution achieves satisfactory fitting to the observed data without embracing high model variance or unconstrained model complexity. Although there is actually more than one solution within the circled corner on the trade-off curve (Fig. 2), the fact that the differences in the current structure revealed from all these solutions are negligible will suffice for our purposes. As indicated in Fig. 2, the variance reductions saturate at about $70 \%$ for the $x$ direction (normal to the coast) and around 90\% for the $y$ direction (along the coastline), respectively. It is obvious that any solution obtained with a damping value lighter than the optimal solution will not achieve significant improvements on the data fitting, but the model variance might increase by several orders of magnitude. On the other hand, heavier damping will further decrease the model variance, but the side effect is that the variance reduction also plunges down rapidly.

The map view pattern of the interpolated mean flow as well as the tidal ellipses for both the M2 and the K1 tides of one of the four layers (at the depth range of $15 \sim 30 \mathrm{~m}$ ) are shown in Fig. 3. It is clear that the dominant transportation is in the along-coast direction. Significant vertical variations across the depth direction are implied from the interpolated result (Figs. 4a,b). Furthermore, the interpolated surface mean flow is compared with results obtained through bin averaging (Fig. 4c) and the RBF expansion (Fig. 4d). There is an obvious resemblance between the results from the RBF expansion (Fig. 4d) and from the multiresolution interpolation (Fig. 4a). However, it is also obvious that the RBF results are characterized by a uniform smoothness, which is a manifestation of the imposed correlation length of the chosen RBF. On the other hand, there is no specific, a priori choice imposed along with the multiresolution approach, and it acquires the final, seemingly smooth, result by accommodating through the resolvable scale hierarchy determined by the local sampling. To avoid leaving out useful information, one possible strategy of undertaking the fitting by the RBF expansion is to start with a sparse grid using an RBF with long correlation length (e.g., long $\sigma_{x}, \sigma_{y}, \sigma_{z}$ for a Gaussian function in 3D space). This is then followed 
(a)

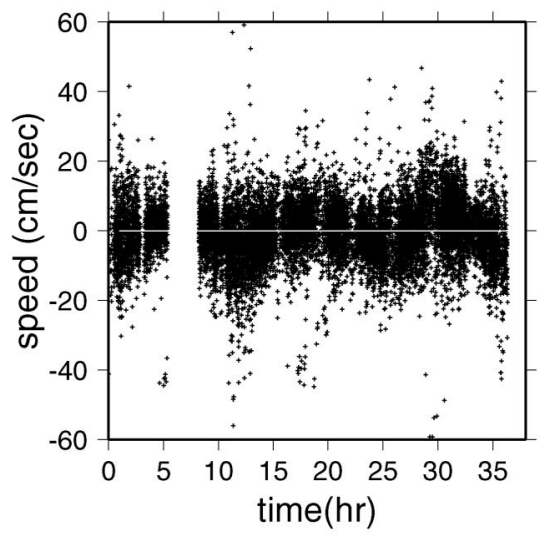

(c)

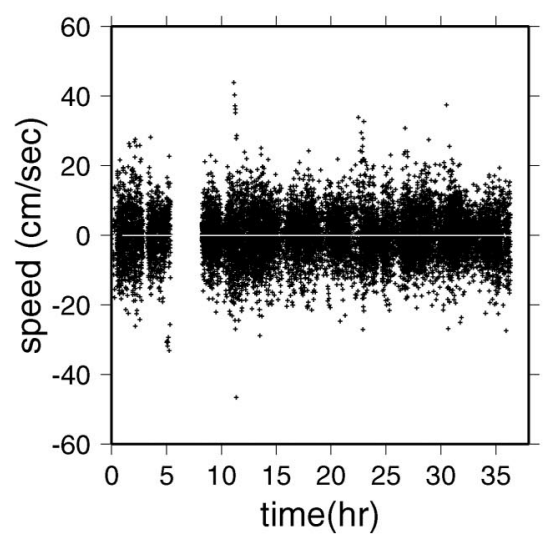

(b)

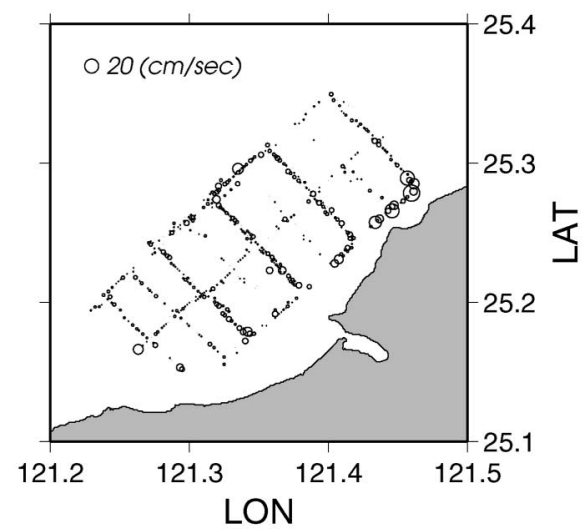

(d)

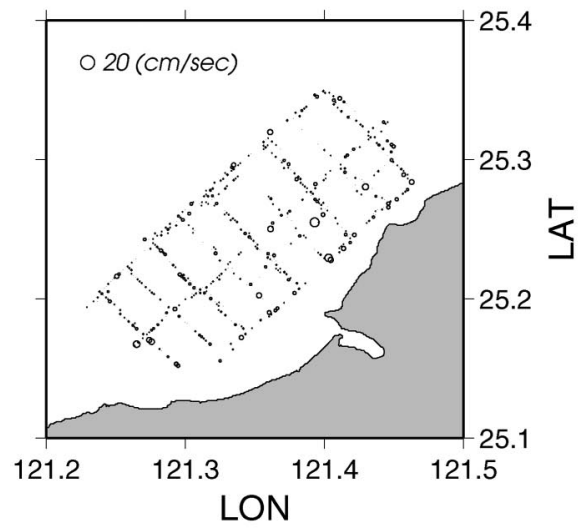

FIG. 6. Distribution of fitting residuals through time (a), (c) irrespective of the spatial locations and (b), (d) across the study region. The upper row $[(a),(b)]$ is for the alongshore $v$ component, whereas the lower row $[(\mathrm{c}),(\mathrm{d})]$ is for the across-shore $u$ component.

by gradually shortening the grid intervals and the correlation lengths of the RBF until the obtained current model does not gain significant additional details. This approach will be analogous to the multigrid algorithm in numerical modeling (e.g., Wesseling 1991). Our multiscale parameterization, however, handles the partitioning among different scales simultaneously due to the multiresolution representation of the current field.

The time evolution of the surface tidal flow is also depicted in Fig. 5. To examine the data fitting, the residuals across the survey duration (Figs. 6a,c) as well as those distributed within the survey area (Figs. 6b,d) are also displayed. The root-mean-square fitting residuals for both directions are 7 and $6 \mathrm{~cm} \mathrm{~s}^{-1}$, respectively. Although there have not been detailed studies undertaken in the same area, there are two recent tide models built for the Taiwan Strait including our surveyed area that can be used for comparison. Since our complete study region appears to be one single point within both the barotropic model of Jan et al. (2001), and the historically averaged low-resolution model of Wang et al.
(2003), we take the spatial median of each of the vertically averaged current fields of our results and compare them to predictions made by these models. The comparison indicates that our median tidal flow is consistent with the tidal flow, with a $81.6 \mathrm{~cm} \mathrm{~s}^{-1}$ major axis and $8.51 \mathrm{~cm} \mathrm{~s}^{-1}$ minor axis, interpolated by Wang et al. (2003) at this area, which is also compatible with the prediction made by the barotropic model of Jan et al. (2001). The median speed of our mean flow, however, is slightly higher, $24 \mathrm{~cm} \mathrm{~s}^{-1}$, as compared to the $16 \mathrm{~cm}$ $\mathrm{s}^{-1}$ speed predicted by Wang et al. (2003). Furthermore, the relative amplitude spectrum of the mean and the M2 and $\mathrm{K} 1$ tides is also compared to the harmonics obtained from a sea level observation within our study region and appears to be consistent.

\section{b. Test example}

To further appraise the performance of the multiresolution detiding scheme, it is desirable to test the method on an artificial dataset with known spatial and transient 
(a) mean

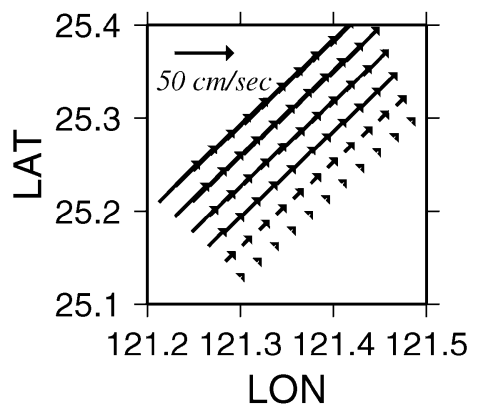

(d) Multi_mean

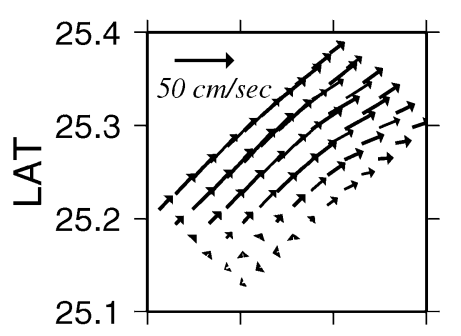

121.2121 .3121 .4121 .5

LON (g) RBF_mean

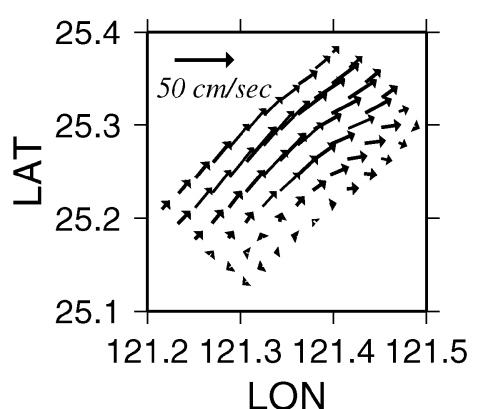

(b) $\mathrm{M} 2$

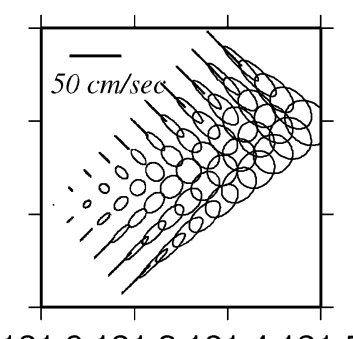

121.2121 .3121 .4121 .5 LON

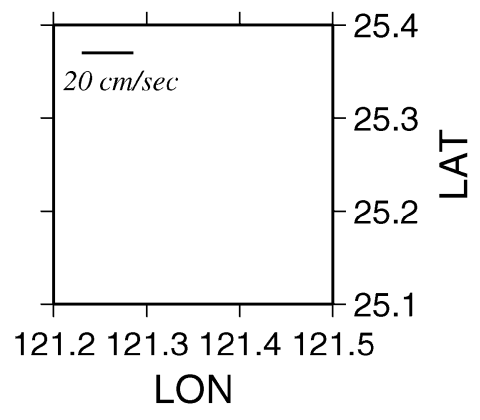

(f) Multi_K1

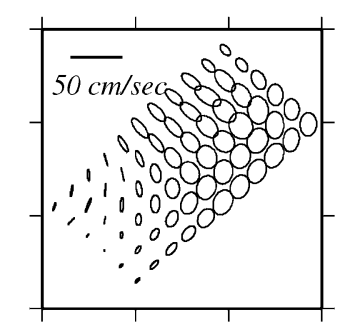

121.2121 .3121 .4121 .5 LON
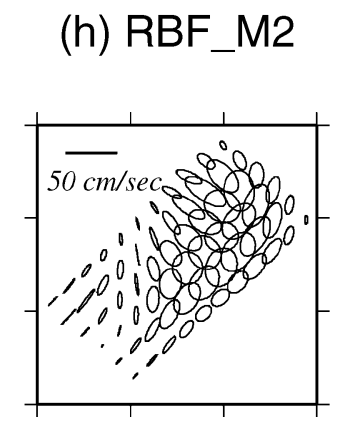

121.2121 .3121 .4121 .5

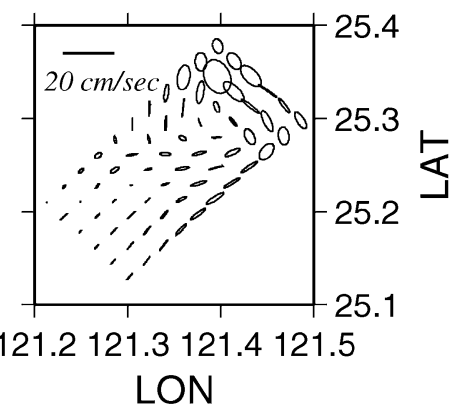

(i) $\mathrm{RBF} \_\mathrm{K} 1$

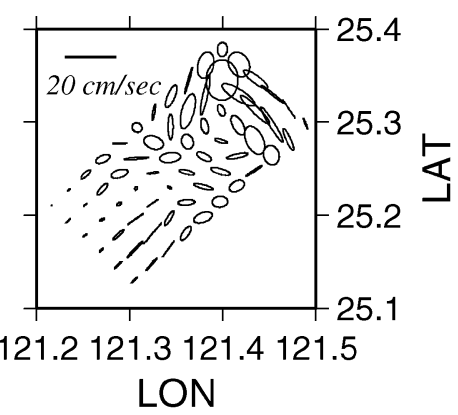

FIG. 7. Interpolated results of the test example. (a)-(c) Plots of the artificial flow field (without the implanted noise), which comprises a smooth mean flow with the linear across-shore speed variation and a simple M2 pattern. (d)-(f) Fitted results obtained with the multiresolution parameterization. (g)-(i) Results from the RBF expansion.

structures so that the results can be compared. The artificial data adopt the identical sampling portrayed by the field data (Fig. 3a), whereas the synthesized current consists in a simple along-coast mean field with linear across-shore speed variation and an M2 tide, also with noticeable spatial variation (Figs. 7a,b). There is also random noise of about $10 \%$ of the peak value of the imposed amplitude of the flow field added to the arti- ficial data. Specifically, as the peak speed of the mean flow and the M2 tide are both set at $50 \mathrm{~cm} \mathrm{~s}^{-1}$, their spatial means amount to 25 and $15 \mathrm{~cm} \mathrm{~s}^{-1}$, respectively. Observation errors are then simulated by adding random numbers within the range of $10 \mathrm{~cm} \mathrm{~s}^{-1}$ to the observations. Since the sampling is the same with the field data, there is no need to perform the trade-off analysis again to determine the damping factor, because the rigor 
(a) mean

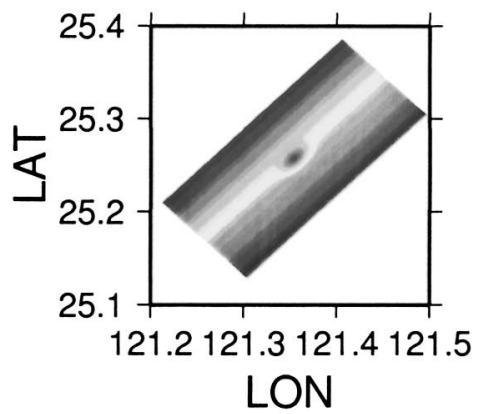

(d) Multi_mean

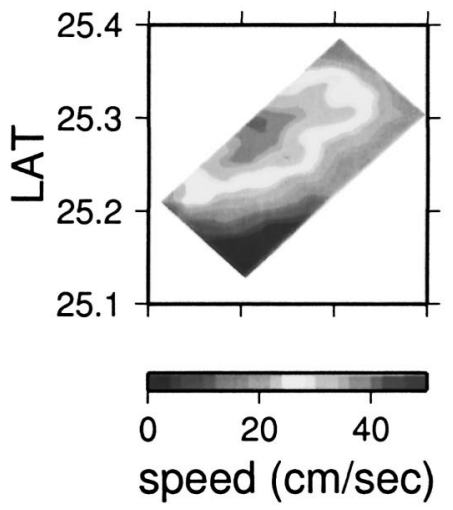

(g) RBF_mean

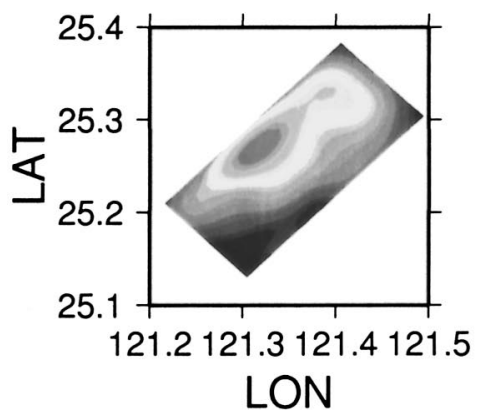

(b) $\mathrm{M} 2$

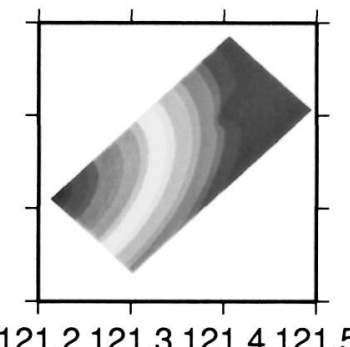

LON

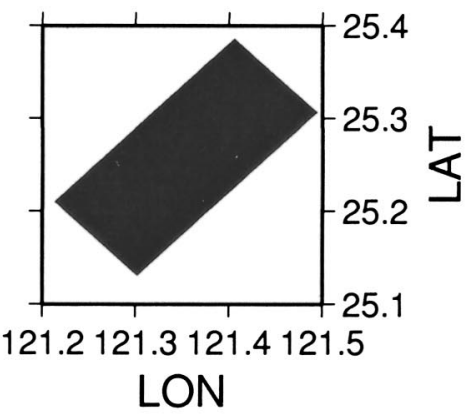

(f) Multi_K1
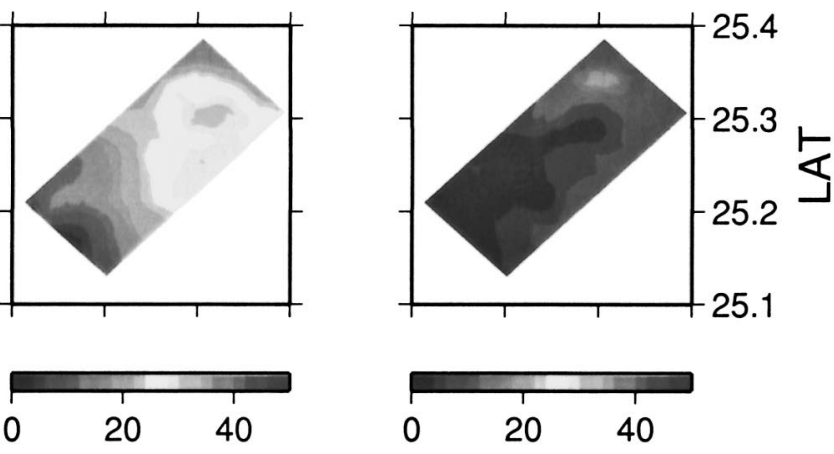

(h) RBF_M2
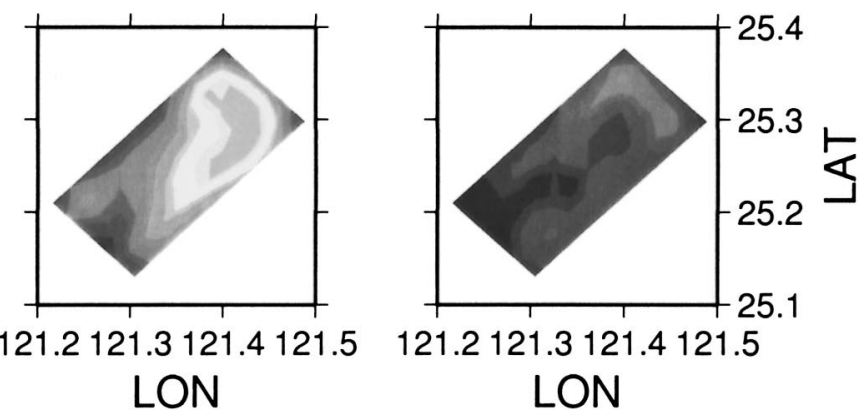

FIG. 8. Similar to Fig. 7 except that the speed contours are presented instead of the velocity vectors and the tidal ellipses.

of the applied damping depends mainly on the sampling. The fitted result indicates an acceptable description of the mean field (Fig. 7d) as compared to the assumed reference field (Fig. 7a). The pattern of the M2 tide is also approximately reconstructed (cf. Fig. 7e with Fig. $7 b)$. It is clear that while variations in the well-sampled area are recovered rather well, only large-scale com- ponents are recovered within regions relatively far away from ship tracks. However, there is also an apparent K1 component within the fitted result (Fig. 7f) that should be absent, since no K1 component is imposed within the artificial data. The obtained K1 component is speculated to be a manifestation of the imperfect sampling as well as the significant level of implanted noise. In a 
supplementary experiment, we find that the amplitude of the obtained K1 component increases significantly with an even higher implanted noise level. Furthermore, it should also be remembered that it is, in general, difficult to actually detide a dataset that lasts for only $36 \mathrm{~h}$, unless we have a priori knowledge that the tidal spectrum is strongly dominated by the M2 field. The results obtained via the RBF expansion, instead of the proposed multiresolution parameterization, are similar but seem to be noisier (Figs. 7g-i). To further clarify the comparisons just described, we also examined the amplitude of the current from the imposed structure (Figs. 8a-c), the results from fitting with the multiresolution parameterization (Figs. 8d-f), and the results from the RBF expansion (Figs. 8g-i). It is apparent that the ghost $\mathrm{K} 1$ components embedded within both the fitting results (Figs. 8f,i) are only slightly above the implanted noise level, mostly well below $15 \mathrm{~cm} \mathrm{~s}^{-1}$. Furthermore, it is also clearly implicated from these reconstructed patterns of amplitude that the sampling of our survey (Fig. 3a) is capable of recovering only the relatively large-scale structures in general.

\section{Discussion and concluding remarks}

Systematic interpolation and detiding of ADCP data is an important challenge for effective and efficient mapping of the coastal currents. Due to the highly uneven sampling, the resolvable current structures are not likely to have a spatially uniform scale spectrum. To extract structures of different scales at different locations according to the available data constraints should be a major concern. In this respect, the proposed multiresolution detiding scheme is a natural data-adaptive scheme that keeps the complete structure of the space-scale hierarchy in the parameterization. The resolvable scales that vary spatially are sorted by the rigor of regularization that sets a threshold on the robustness of the obtained model parameters. In essence, it automatically adjusts the adequate scale bandwidths that have enough samples suitable to be processed by the harmonic analysis, such that the local mean field and tidal fields can be separated at different scales. In contrast, the binaveraging scheme has only a fixed characteristic scale set up by the grid intervals. Interpolation schemes invoking biharmonic splines or other RBF types of interpolation functions have a priori scale bandwidths that are inherited from the particular chosen functions and are irrespective of the data constraints. In this sense, the multiresolution scheme incorporates merits of these two previously popular techniques, which operate at spatially different scales depending upon the local data constraints. It is implemented by the straightforward wavelet transform of the constraining equations in the threedimensional space and is numerically efficient.

Acknowledgments. Comments from two anonymous reviewers helped clarify and improve the article signif- icantly. All figures were prepared using GMT software version 3.0 (Wessel and Smith 1991). This work was supported by the National Science Council of the Republic of China, through Grant NSC-90-2611-M-002002.

\section{REFERENCES}

Bijwarrd, H., W. Spakman, and E. R. Engdahl, 1998: Closing the gap between regional and global travel time tomography. J. Geophys. Res., 103, 30 055-30078.

Bogden, P. S., and J. O’Donnell, 1998: Generalized inverse of shipboard current measurements: Tidal and non-tidal flows in Long Island Sound. J. Mar. Res., 56, 995-1027.

Bruce, A., and H.-Y. Gao, 1996: Applied Wavelet Analysis with SPlus. Springer, 338 pp.

Candela, J., R. C. Beardsley, and R. Limeburner, 1990: Removing tides from ship-mounted ADCP data, with application to the Yellow Sea. Proc. IEEE Fourth Working Conf. on Current Measurements, Airlie, VA, Institute of Electrical and Electronics Engineers, 258-266.

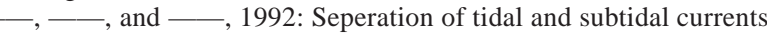
in ship-mounted acoustic Doppler current profiler observations. J. Geophys. Res., 97, 769-788.

Chiao, L.-Y., and B.-Y. Kuo, 2001: Multiscale seismic tomography. Geophys. J. Int., 145, 517-527.

__ , and W.-T. Liang, 2003: Multiresolution parameterization for geophysical inverse problems. Geophysics, 68, 199-209.

Cohen, A., I. Daubechies, and J.-C. Feauveau, 1992: Biorthogonal bases of compactly supported wavelets. Commun. Pure Appl. Math., 45, 485-560.

Dowd, M., and K. R. Thompson, 1996: Extraction of tidal streams from a ship-borne acoustic Doppler current profiler using a statistical-dynamical model. J. Geophys. Res., 101, 8943-8956.

Foreman, M. G. G., and H. J. Freeland, 1991: A comparison of techniques for tide removal from ship-mounted acoustic Doppler measurements along the southwest coast of Vancouver Island. J. Geophys. Res., 96, 17 007-17 021.

Geyer, R., and R. Signell, 1990: Tidal flow measurements around a headland with shipboard acoustic Doppler current profiler. $J$. Geophys. Res., 95, 3189-3197.

Gualtiero, B., and A. L. Vesnaver, 1999: In quest of the grid. Geophysics, 64, 116-1125.

Jan, S., Y.-H. Wang, S.-Y. Chao, and D.-P. Wang, 2001: Development of a nowcast system for the Taiwan Strait. Ocean Polar Res., 22, 195-203.

Kirby, M., 2001: Geometric Data Analysis-An Empirical Approach to Dimensionality Reduction and the Study of Patterns. John Wiley and Sons, $363 \mathrm{pp}$.

Lines, L. R., and S. Treitel, 1983: Tutorial: A review of least-squares inversion and its application to geophysical problems. Geophys. Prospect., 32, 159-186.

Mallat, S., 1989a: A theory of multiresolution signal decomposition: The wavelet representation. IEEE Trans. Pattern An. Mach. Intell., 11, 674-693.

, 1989b: Multiresolution approximations and wavelet orthonormal bases of $L^{2}(R)$. Trans. Amer. Math. Soc., 315, 69-88.

_ 1998: A Wavelet Tour of Signal Processing. Academic Press, $577 \mathrm{pp}$.

Meyerholtz, K. A., G. L. Pavlis, and S. A. Szpakowski, 1989: Convolutional quelling in seismic tomography. Geophysics, 54, 570580 .

Münchow, A., 2000: Detiding three-dimensional velocity survey data in coastal waters. J. Atmos. Oceanic Technol., 17, 736-748.

Paige, C. C., and M. A. Saunders, 1982: LSQR: An algorithm for sparse linear equations and sparse least squares. ACM Trans. Math. Software, 8, 43-71.

Press, W. H., S. A. Teukolsky, W. T. Vetterling, and B. P. Flannery, 
1992: Numerical Recipes in FORTRAN: The Art of Scientific Computing. 2d. ed. Cambridge University Press, 963 pp.

Sandwell, D. T., 1987: Biharmonic spline interpolation of GEOS3 and SEASAT altimeter data. Geophys. Res. Lett., 14, 139142 .

Simpson, J. H., E. G. Mitchelson-Jacob, and A. E. Hill, 1990: Flow structure in a channel from an acoustic Doppler current profiler. Cont. Shelf Res., 10, 589-603.

Tikhonov, A. N., and A. V. Goncharsky, 1987: Ill-posed Problems in the Natural Sciences. Mir Publishers, 344 pp.

Wang, Y.-H., S. Jan, and D.-P. Wang, 2003: Transports through Taiwan Strait from shipboard ADCP observations (19992001). Estuarine Coastal Mar. Sci., 57, 195-201.

Wessel, J. K., and H. F. Smith, 1991: Free software helps map and display data. Eos, Trans. Amer. Geophys. Union, 72, 441, 445446.

Wesseling, P., 1991: An Introduction to Multigrid Methods. John Wiley and Sons, $284 \mathrm{pp}$. 